\title{
Quality of Life in Patients with Knee Osteoarthritis: A Commentary on Nonsurgical and Surgical Treatments
}

\author{
Jack Farr II $^{1}$, Larry E. Miller ${ }^{2,3}$ and Jon E. Block ${ }^{*, 3}$ \\ ${ }^{I}$ OrthoIndy South, 1260 Innovation Pkwy., Suite 100, Greenwood, IN 46143, USA \\ ${ }^{2}$ Miller Scientific Consulting, Inc., 26 Portobello Road, Arden, NC 28704, USA \\ ${ }^{3}$ The Jon Block Group, 2210 Jackson Street, Suite 401, San Francisco, CA 94115, USA
}

\begin{abstract}
Knee osteoarthritis (OA) has a significant negative impact on health-related quality of life (HRQoL). Identification of therapies that improve HRQoL in patients with knee OA may mitigate the clinical, economic, and social burden of this disease. The purpose of this commentary is to report the impact of knee OA on HRQoL, describe the change in HRQoL attributable to common knee OA interventions, and summarize findings from clinical trials of a promising therapy. Nonsurgical therapies do not reliably modify HRQoL in knee OA patients given their general inability to alleviate physical manifestations of OA. Surgical knee OA interventions generally result in good to excellent patient outcomes. However, there are significant barriers to considering surgery, which limits clinical utility. Therapies that most effectively control OA-related pain with a low risk: benefit ratio will likely have the greatest benefit on HRQoL with greater rates of patient adoption. Initial clinical trial findings suggest that less invasive joint unloading implants hold promise in bridging the therapeutic gap between nonsurgical and surgical treatments for the knee OA patient.
\end{abstract}

Keywords: Intervention, knee, osteoarthritis, pain, quality of life, unloading.

\section{INTRODUCTION}

Chronic musculoskeletal conditions are prevalent worldwide and their impact on the individual is significant. At any given time, $30 \%$ of American adults are affected by joint pain, swelling, or limitation of movement [1]. Osteoarthritis (OA), particularly at the knee, is a leading cause of disability in older adults [2] characterized by progressive articular cartilage loss resulting in joint pain and disability [3]. By 2025, the prevalence of knee OA is expected to increase by $40 \%$, largely due to an aging population and the obesity epidemic [1]. The burden of knee OA on individuals, health systems, and social care systems is considered by many as a public health crisis [4].

Limitations in walking, stair climbing, and squatting are common patient complaints that greatly interfere with activities of daily living and recreation. Consequently, most research on knee OA has attempted to quantify the magnitude of physical disability associated with this disease and the impact of various treatments on outcomes such as pain severity and physical functioning. However, the physical manifestations of knee OA have direct impact on other aspects of patient's lives such as social interactions, mental functioning, and sleep quality [5]. Therefore, healthrelated quality of life (HRQoL) measures are relevant and important adjunct outcomes that help quantify the physical, social, and emotional impact of knee OA and of various OA therapies.

*Address correspondence to this author at the Jon Block Group, 2210 Jackson Street, Suite 401, San Francisco, CA 94115, USA; Tel: +1 415775 7947; Fax: +415 928 0765; E-mail: jonblock@jonblockgroup.com
HRQoL is generally considered to comprise five main dimensions including physical functioning, psychological functioning, social functioning, cognitive functioning, and general well-being [6]. Explicit in the framework of HRQoL is the evaluation of functional status as well as patient perceptions of emotional and social functioning and role activities. This concept is critical since patients vary greatly in their responses to identical stressors, such as pain. Not only does HRQoL offer a more holistic approach to quantifying patient experiences compared to isolated, disease-specific outcomes, but HRQoL measures also have direct relevance to clinical practice since they are highly predictive of mortality and health care resource utilization [7-9].

Compared with other chronic diseases, patients with musculoskeletal disorders report among the lowest HRQoL, with knee OA patients reporting lower scores on every HRQoL parameter compared to age-matched norms [10]. Not surprisingly, HRQoL progressively declines in knee OA patients, concomitant with the magnitude of disease progression [11, 12]. Identification of therapies that improve HRQoL in patients with knee OA may mitigate the clinical, economic, and social burden of this disease. The purpose of this commentary was to report the impact of knee OA on HRQoL, describe the change in HRQoL attributable to common knee OA interventions, and summarize findings from clinical trials of a promising therapy.

\section{HRQOL IN KNEE OSTEOARTHRITIS PATIENTS}

A large body of evidence substantiates lower HRQoL scores in knee OA patients compared to age-matched norms. Increasing joint pain comorbidity [12] and radiographic 
disease severity [13] are inversely related to HRQoL in knee OA patients. As the disease progresses, HRQoL declines to levels significantly below that of the general population. Desmeules et al. [14] reported mean Physical Component Summary (PCS) and Mental Component Summary (MCS) scores from the SF-36 of 28 and 43, respectively, in 197 patients waiting for total knee arthroplasty (TKA); both of these values are well below the $25^{\text {th }}$ percentile compared to age- and gender-matched normative values [15]. Other studies have reported similarly low HRQoL in patients waiting for TKA [11, 16]. Notably, within a group of patients waiting for TKA who already report very low HRQoL, nearly 3 in 4 reported continued worsening in HRQoL during the mean 10-month waiting period before surgery [17]. Even more concerning is that since less than one-third of patients with severe knee $\mathrm{OA}$ are willing to undergo arthroplasty [18], it is reasonable to assume that most patients with severe knee OA suffer low HRQoL throughout the remainder of life.

\section{EFFECT OF NONSURGICAL INTERVENTIONS ON HRQOL}

The effectiveness of nonsurgical knee OA interventions in alleviating pain and improving joint function is generally unsatisfactory and transient [19]. Concomitant with the lack of meaningful change in self-reported symptoms, HRQoL changes with nonsurgical treatment are modest. Consequently, there is a growing consensus that most nonsurgical knee OA treatments are misguided [19]. Although the etiology of knee OA is multifactoral, chronic aberrant and excessive knee joint loading are arguably the primary risk factors [20, 21]. In general, conservative therapies that fail to address the underlying mechanism of disease have poor efficacy and those that do impact joint loading biomechanics are associated with poor long-term patient adherence.

Regular physical activity, especially low-impact aerobic exercise and quadriceps strengthening programs, are strongly encouraged in the knee OA patient [22]. Despite this recommendation, the evidence supporting physical activity participation on improving HRQoL is limited, variable, and highly dependent on the exercise modality. Bennell and colleagues [23] reported that HRQoL was no different in patients undergoing a 12-week multi-modal physiotherapy program compared to those receiving sham ultrasound. Brosseau and colleagues [24] randomized knee OA patients to walking with behavior intervention, walking with an educational pamphlet, or pamphlet alone. No changes in HRQoL were observed in any of these groups during the 1year intervention period. Evcik and Sonel [25] reported greater HRQoL improvements with a 3-month group walking program compared to home-based exercise, despite the fact that pain and function scores were similar between the groups. The components of HRQoL attributable for the global HRQoL improvement in the walking group included sleep quality, emotional reactions, physical mobility, and energy. Kao and coworkers [26] reported no changes in HRQoL with self-management consisting of patient education and exercise or usual care for 8 weeks. However, a similar 6-month disease-specific self-management program improved HRQoL $15-20 \%$ in domains related to pain and physical function [27]. Tai chi has been reported to modestly
(20\%) improve PCS, but not MCS scores [28, 29]. Finally, yoga therapy has consistently been shown to improve physical and emotional HRQoL parameters [30]. Overall, physical activity programs appear to, at best, modestly improve physical domains of HRQoL with minimal impact on the mental domains in knee OA patients. The efficacy of physical activity on HRQoL in patients with knee OA may be limited by the well-known problem of poor adherence to exercise programs in this patient population [31].

Oral analgesic and anti-inflammatory agents are perhaps the most commonly prescribed treatments to alleviate knee OA symptoms with almost 3 in 4 patients reporting regular use [32]. Despite this widespread use, the clinical effectiveness of these agents is limited [33]. Short-term administration of rofecoxib [34], celecoxib [35], and lidocaine patch [35] has yielded variable results on HRQoL. No known studies have examined HRQoL with long-term use of analgesics or non-steroidal anti-inflammatory drugs. The study with the longest follow-up duration reported that analgesic use had no impact on PCS or MCS through 1 year of use [36]. Regular use of these agents has no impact on disease progression and, in fact, they may accelerate OA progression due to higher resulting forces across the knee joint [37-40]. Therefore, their long-term benefit on HRQoL is doubtful.

Lateral wedge insoles and varus- or valgus-knee braces are mainstay first-line treatments in the physician's armatorium of OA therapies. Although the premise behind redistributing the forces about the knee joint is sound, the clinical utility of these therapies is limited mainly by patient discomfort and resulting poor adherence to therapy. Neither lateral wedge insoles [41-44] nor knee bracing [45, 46] provides demonstrable clinical benefit on knee pain or disease progression. Bennell and colleagues [44] reported that HRQoL was no different in patients with lateral wedge insoles compared to those receiving flat control insoles. Skou and coworkers [47] were able to demonstrate modest HRQoL improvements, but only after excluding the almost $20 \%$ of patients who withdrew from the study. Brouwer et al. [48] randomized 117 patients with knee OA to a knee brace and standard of care or standard of care alone. These authors reported that the addition of the knee brace had no effect on HRQoL. The totality of evidence on these joint offloading therapies is not supportive of their use either to relieve symptoms or to improve HRQoL, with patient compliance issues likely limiting the responsiveness of these outcomes.

The use of viscosupplementation in knee OA treatment generally improves HRQoL parameters, although the clinical effects generally subside after 3 to 6 months. Maheu et al. [49] reported $27 \%$ improvements in PCS, but only $4 \%$ improvements in MCS at 24 weeks following hyaluronic acid injection. Most studies of viscosupplements have reported modest changes in selected components of HRQoL (mainly physical), but not in global scores [50]. Although patients may undergo repeat injections in an effort to maintain tolerable pain levels and acceptable HRQoL, the beneficial impact of viscosupplements on HRQoL decreases with repeated injections [51]. Additionally, the changes in functional pain scores with viscosupplementation do not correlate to quality of life scores [50]. Similar to findings 
with acetaminophen and NSAIDS, hyaluronic acid injections may actually increase knee joint loading and accelerate joint deterioration due to short-term pain amelioration [37]. Finally, the safety of HA injections has recently been questioned [52]. Overall, hyaluronic acid injections are ineffective in durably increasing $\mathrm{HRQ} o \mathrm{~L}$ in the knee OA patient.

\section{EFFECT OF SURGICAL INTERVENTIONS ON HRQOL}

Surgical repair of symptomatic knee OA is widely utilized, with over 600,000 cases performed annually in the US [53]. High tibial osteotomy (HTO), unicompartmental knee arthroplasty (UKA), and TKA each reliably restore physical function and alleviate pain [54]. Additionally, the HRQoL improvements observed following these surgeries are similarly impressive. Several studies have reported HRQoL increases of at least $100 \%$ through 2 to 7 years following TKA, UKA, or HTO [16, 55-60]. However, some suggest that postoperative HRQoL improvements following TKA are not durable, with declines identified around 3 years post-surgery [61].

A major and often overlooked consideration when evaluating $\mathrm{OA}$ therapies is patient willingness to undergo a particular treatment, regardless of therapeutic value. For example, TKA is well-known to substantially improve OArelated pain and function in a cost effective manner [62]. However, less than one-third of TKA candidates are actually willing to undergo the surgery [18], due in large part to lack of confidence in beneficial surgery outcomes and high operative risk due to presence of multiple comorbidities [18]. Additionally, each of these surgical options permanently modifies the knee joint via an invasive, irreversible surgical procedure, which may also negatively impact patient willingness to undergo these procedures.

\section{FUTURE DIRECTIONS}

For unicompartmental knee OA, the optimal surgery would result in similar safety and effectiveness outcomes as HTO and arthroplasty, but would also enjoy high patient acceptance rates, thereby allowing greater utilization by symptomatic patients. The most common unicompartmental knee OA presentation is medial typically associated with varus alignment. Extra-capsular medial compartment knee load absorber implants that require only two incisions for implant are currently under evaluation (Fig. 1). Three clinical trials have been completed with these implants, with each reporting impressive improvements in HRQoL. The OASYS study (ACTRN12608000451303) treated 30 patients with this knee joint unloading implant. At 3 years, PCS increased from $36 \pm 9$ at pre-treatment to $52 \pm 5$ $(\mathrm{p}<0.001)$, a value higher than age-matched norms and comparable to values seen following TKA. No changes in MCS were noted $(56 \pm 8$ at baseline and at 3 years). The OAKS study $(\mathrm{n}=30)$ (ACTRN12609001068257) reported KOOS QOL domain scores improvements from $20 \pm 18$ to $52 \pm 18(\mathrm{p}<0.001)$ at 1 year. These results were confirmed in the COAST study of 40 patients (ISRCTN63048529), which demonstrated improvements in the KOOS QOL domain 1 year following treatment from $22 \pm 15$ to $51 \pm 23(\mathrm{p}<0.001)$. Importantly, these KOOS HRQoL changes well exceeded the minimal clinically important difference of 21 points established for knee OA patients [63] and were at least comparable to HRQoL changes observed with TKA, UKA, and HTO. Larger-scale clinical trials with these joint unloading implants are required, but initial results are promising and suggest utility in improving HRQoL in knee OA patients.

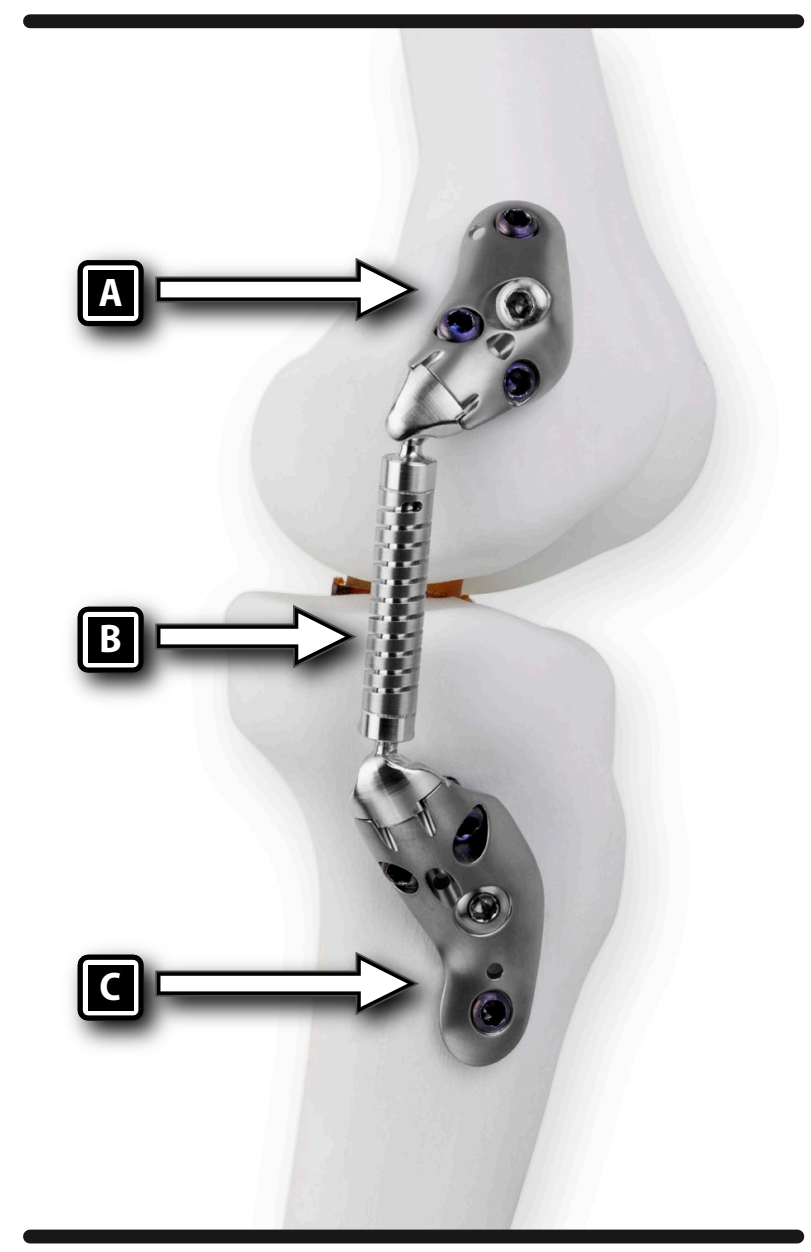

Fig. (1). Medial view of an extra-capsular medial compartment knee load absorber implant. a) femoral base, b) absorber unit, c) tibial base.

\section{CONCLUSIONS}

A primary objective of health and medical care is to enhance quality of life, independent of alleviating diseaserelated symptoms. The evaluation of new therapeutic interventions for knee $\mathrm{OA}$ requires a thorough understanding of the impact of a treatment on a patient's physical, social, and psychological status. Therapies for knee OA should ideally decrease pain, improve physical functioning, and increase HRQoL in a safe and durable manner. The failure of nonsurgical therapies to modify HRQoL in knee OA patients is not surprising given their inability to alleviate physical manifestations of OA. Surgical knee OA interventions generally result in good to excellent patient outcomes. However, there are significant barriers to considering surgery, which limits clinical utility. Since the strongest correlate of global measures of HRQoL in the OA patient is pain severity [64], therapies that most effectively control 
OA-related pain while imparting no negative consequences (e.g. high cost, side effects, invasive surgery, etc.) will likely have the greatest benefit on HRQoL with greater rates of patient adoption. Less invasive joint unloading implants hold promise in bridging this therapeutic gap for the patient with medial compartment knee OA.

\section{CONFLICT OF INTEREST}

LEM and JEB are consultants to Moximed, Inc. (Hayward, CA, USA).

\section{ACKNOWLEDGEMENTS}

Declared none.

\section{REFERENCES}

[1] Woolf AD, Pfleger B. Burden of major musculoskeletal conditions. Bull World Health Organ 2003; 81(9): 646-56.

[2] Murphy L, Helmick CG. The impact of osteoarthritis in the United States: a population-health perspective: A population-based review of the fourth most common cause of hospitalization in U.S. adults. Orthop Nurs 2012; 31(2): 85-91.

[3] Martel-Pelletier J, Boileau C, Pelletier JP, Roughley PJ. Cartilage in normal and osteoarthritis conditions. Best Pract Res Clin Rheumatol 2008; 22(2): 351-84

[4] White $\mathrm{PH}$, Waterman M. Making osteoarthritis a public health priority. Am J Nurs 2012; 112(3 Suppl 1): S20-5.

[5] Ferrell BA. Pain management in elderly people. J Am Geriatr Soc 1991; 39(1): 64-73.

[6] Revicki DA. Health-related quality of life in the evaluation of medical therapy for chronic illness. J Fam Pract 1989; 29(4): 37780.

[7] Dorr DA, Jones SS, Burns L, et al. Use of health-related, qualityof-life metrics to predict mortality and hospitalizations in community-dwelling seniors. J Am Geriatr Soc 2006; 54(4): 66773.

[8] Singh JA, Nelson DB, Fink HA, Nichol KL. Health-related quality of life predicts future health care utilization and mortality in veterans with self-reported physician-diagnosed arthritis: the veterans arthritis quality of life study. Semin Arthritis Rheum 2005; 34(5): 755-65.

[9] Singh JA, Borowsky SJ, Nugent S, et al. Health-related quality of life, functional impairment, and healthcare utilization by veterans: veterans' quality of life study. J Am Geriatr Soc 2005; 53(1): 10813.

[10] Picavet HS, Hoeymans N. Health related quality of life in multiple musculoskeletal diseases: SF-36 and EQ-5D in the DMC3 study. Ann Rheum Dis 2004; 63(6): 723-9.

[11] Snider MG, MacDonald SJ, Pototschnik R. Waiting times and patient perspectives for total hip and knee arthroplasty in rural and urban Ontario. Can J Surg 2005; 48(5): 355-60.

[12] Hoogeboom TJ, den Broeder AA, de Bie RA, van den Ende CH. Longitudinal impact of joint pain comorbidity on quality of life and activity levels in knee osteoarthritis: data from the Osteoarthritis Initiative. Rheumatology (Oxford) 2013; 52(3): 543-6.

[13] Muraki S, Akune T, Oka H, et al. Association of radiographic and symptomatic knee osteoarthritis with health-related quality of life in a population-based cohort study in Japan: the ROAD study. Osteoarthritis Cartil 2010; 18(9): 1227-34.

[14] Desmeules F, Dionne CE, Belzile E, Bourbonnais R, Fremont P. Waiting for total knee replacement surgery: factors associated with pain, stiffness, function and quality of life. BMC Musculoskelet Disord 2009; 10: 52.

[15] Ware JE, Kosinski M, Dewey JE. How to score version 2 of the SF-36 Health Survey. Lincoln, RI: QualityMetric Incorporated 2000.

[16] Tsonga T, Kapetanakis S, Papadopoulos C, et al. Evaluation of improvement in quality of life and physical activity after total knee arthroplasty in greek elderly women. Open Orthop J 2011; 5: 3437.

[17] Ackerman IN, Bennell KL, Osborne RH. Decline in Health-Related Quality of Life reported by more than half of those waiting for joint replacement surgery: a prospective cohort study. BMC Musculoskelet Disord 2011; 12: 108.
[18] Ballantyne PJ, Gignac MA, Hawker GA. A patient-centered perspective on surgery avoidance for hip or knee arthritis: lessons for the future. Arthritis Rheum 2007; 57(1): 27-34.

[19] Crawford DC, Miller LE, Block JE. Conservative management of symptomatic knee osteoarthritis: a flawed strategy? Orthop Rev 2013; 5(1): 5-10.

[20] Waller C, Hayes D, Block JE, London NJ. Unload it: the key to the treatment of knee osteoarthritis. Knee Surg Sports Traumatol Arthrose 2011; 19(11): 1823-9.

[21] Brandt KD, Dieppe P, Radin E. Etiopathogenesis of osteoarthritis. Med Clin North Am 2009; 93(1): 1-24, xv.

[22] Richmond J, Hunter D, Irrgang J, et al. Treatment of osteoarthritis of the knee (nonarthroplasty). J Am Acad Orthop Surg 2009; 17(9): 591-600.

[23] Bennell KL, Hinman RS, Metcalf BR, et al. Efficacy of physiotherapy management of knee joint osteoarthritis: a randomised, double blind, placebo controlled trial. Ann Rheum Dis 2005; 64(6): 906-12.

[24] Brosseau L, Wells GA, Kenny GP, et al. The implementation of a community-based aerobic walking program for mild to moderate knee osteoarthritis: a knowledge translation randomized controlled trial: part II: clinical outcomes. BMC Public Health 2012; 12: 1073.

[25] Evcik D, Sonel B. Effectiveness of a home-based exercise therapy and walking program on osteoarthritis of the knee. Rheumatol Int 2002; 22(3): 103-6.

[26] Kao MJ, Wu MP, Tsai MW, Chang WW, Wu SF. The effectiveness of a self-management program on quality of life for knee osteoarthritis (OA) patients. Arch Gerontol Geriatr 2012; 54(2): 317-24.

[27] Coleman S, Briffa NK, Carroll G, Inderjeeth C, Cook N, McQuade J. A randomised controlled trial of a self-management education program for osteoarthritis of the knee delivered by health care professionals. Arthritis Res Ther 2012; 14(1): R21.

[28] Wang C, Schmid CH, Hibberd PL, et al. Tai Chi is effective in treating knee osteoarthritis: a randomized controlled trial. Arthritis Rheum 2009; 61(11): 1545-53.

[29] Lee HJ, Park HJ, Chae Y, et al. Tai Chi Qigong for the quality of life of patients with knee osteoarthritis: a pilot, randomized, waiting list controlled trial. Clin Rehabil 2009; 23(6): 504-11.

[30] Ebnezar J, Nagarathna R, Bali Y, Nagendra HR. Effect of an integrated approach of yoga therapy on quality of life in osteoarthritis of the knee joint: A randomized control study. Int J Yoga 2011; 4(2): 55-63.

[31] Marks R. Knee osteoarthritis and exercise adherence: a review. Curr Aging Sci 2012; 5(1): 72-83.

[32] Dillon CF, Rasch EK, Gu Q, Hirsch R. Prevalence of knee osteoarthritis in the United States: arthritis data from the Third National Health and Nutrition Examination Survey 1991-94. J Rheumatol 2006; 33(11): 2271-9.

[33] Scott DL, Berry H, Capell H, et al. The long-term effects of nonsteroidal anti-inflammatory drugs in osteoarthritis of the knee: a randomized placebo-controlled trial. Rheumatology (Oxford) 2000; 39(10): 1095-101.

[34] Theiler R, Bischoff HA, Good M, Uebelhart D. Rofecoxib improves quality of life in patients with hip or knee osteoarthritis. Swiss Med Wkly 2002; 132(39-40): 566-73.

[35] Kivitz A, Fairfax M, Sheldon EA, et al. Comparison of the effectiveness and tolerability of lidocaine patch $5 \%$ versus celecoxib for osteoarthritis-related knee pain: post hoc analysis of a 12 week, prospective, randomized, active-controlled, open-label, parallel-group trial in adults. Clin Ther 2008; 30(12): 2366-77.

[36] Mody S, Jolly M, Kwasny MJ, Block JA. Patient reported outcomes and analgesia use in osteoarthritis of the knee. Osteoarthritis Cartil 2008; 16(11): 1294-9.

[37] Briem K, Axe MJ, Snyder-Mackler L. Medial knee joint loading increases in those who respond to hyaluronan injection for medial knee osteoarthritis. J Orthop Res 2009; 27(11): 1420-5.

[38] Henriksen M, Simonsen EB, Alkjaer T, et al. Increased joint loads during walking--a consequence of pain relief in knee osteoarthritis. Knee 2006; 13(6): 445-50.

[39] Hurwitz DE, Sharma L, Andriacchi TP. Effect of knee pain on joint loading in patients with osteoarthritis. Curr Opin Rheumatol 1999; 11(5): 422-6.

[40] Ding C, Cicuttini F, Jones G. Do NSAIDs affect longitudinal changes in knee cartilage volume and knee cartilage defects in older adults? Am J Med 2009; 122(9): 836-42. 
[41] Baker K, Goggins J, Xie H, et al. A randomized crossover trial of a wedged insole for treatment of knee osteoarthritis. Arthritis Rheum 2007; 56(4): 1198-203.

[42] Maillefert JF, Hudry C, Baron G, et al. Laterally elevated wedged insoles in the treatment of medial knee osteoarthritis: a prospective randomized controlled study. Osteoarthritis Cartil 2001; 9(8): 73845.

[43] Pham T, Maillefert JF, Hudry C, et al. Laterally elevated wedged insoles in the treatment of medial knee osteoarthritis. A two-year prospective randomized controlled study. Osteoarthritis Cartil 2004; 12(1): 46-55.

[44] Bennell KL, Bowles KA, Payne C, et al. Lateral wedge insoles for medial knee osteoarthritis: 12 month randomised controlled trial. BMJ 2011; 342: d2912.

[45] Warden SJ, Hinman RS, Watson MA, Jr., Avin KG, Bialocerkowski AE, Crossley KM. Patellar taping and bracing for the treatment of chronic knee pain: a systematic review and metaanalysis. Arthritis Rheum 2008; 59(1): 73-83.

[46] Brouwer RW, Jakma TS, Verhagen AP, Verhaar JA, BiermaZeinstra SM. Braces and orthoses for treating osteoarthritis of the knee. Cochrane Database Syst Rev 2005(1): CD004020.

[47] Skou ST, Hojgaard L, Simonsen OH. Customized foot insoles have a positive effect on pain, function, and quality of life in patients with medial knee osteoarthritis. J Am Podiatr Med Assoc 2013; 103(1): 50-5.

[48] Brouwer RW, van Raaij TM, Verhaar JA, Coene LN, BiermaZeinstra SM. Brace treatment for osteoarthritis of the knee: a prospective randomized multi-centre trial. Osteoarthritis Cartil 2006; 14(8): 777-83.

[49] Maheu E, Zaim M, Appelboom T, et al. Comparative efficacy and safety of two different molecular weight (MW) hyaluronans F60027 and Hylan G-F20 in symptomatic osteoarthritis of the knee (KOA). Results of a non inferiority, prospective, randomized, controlled trial. Clin Exp Rheumatol 2011; 29(3): 527-35.

[50] Vincent HK, Montero C, Conrad BP, et al. "Functional pain," functional outcomes, and quality of life after hyaluronic acid intraarticular injection for knee osteoarthritis. PM R 2013; 5(4): 310-8.

[51] Rat AC, Baumann C, Guillemin F. National, multicentre, prospective study of quality of life in patients with osteoarthritis of the knee treated with hylane G-F 20. Clin Rheumatol 2011; 30(10): 1285-93.

[52] Rutjes AW, Juni P, da Costa BR, Trelle S, Nuesch E, Reichenbach S. Viscosupplementation for osteoarthritis of the knee: a systematic review and meta-analysis. Ann Intern Med 2012; 157(3): 180-91.
[53] Agency for Healthcare Research and Quality. Healthcare Cost and Utilization Project. 2010; Available from: http: //hcupnet.ahrq.gov/ HCUPnet.jsp

[54] Zhang W, Moskowitz RW, Nuki G, et al. OARSI recommendations for the management of hip and knee osteoarthritis, Part II: OARSI evidence-based, expert consensus guidelines. Osteoarthritis Cartil 2008; 16(2): 137-62.

[55] Birmingham TB, Giffin JR, Chesworth BM, et al. Medial opening wedge high tibial osteotomy: a prospective cohort study of gait, radiographic, and patient-reported outcomes. Arthritis Rheum 2009; 61(5): 648-57.

[56] Argenson JN, Parratte S, Ashour A, Komistek RD, Scuderi GR. Patient-reported outcome correlates with knee function after a single-design mobile-bearing TKA. Clin Orthop Relat Res 2008; 466(11): 2669-76.

[57] Ko Y, Narayanasamy S, Wee HL, et al. Health-related quality of life after total knee replacement or unicompartmental knee arthroplasty in an urban asian population. Value Health 2011; 14(2): $322-8$

[58] Roos EM, Toksvig-Larsen S. Knee injury and Osteoarthritis Outcome Score (KOOS) - validation and comparison to the WOMAC in total knee replacement. Health Qual Life Outcomes 2003; 1: 17.

[59] Bachmeier CJ, March LM, Cross MJ, et al. A comparison of outcomes in osteoarthritis patients undergoing total hip and knee replacement surgery. Osteoarthritis Cartil 2001; 9(2): 137-46.

[60] Dahl WA, Toksvig-Larsen S, Roos EM. A 2-year prospective study of patient-relevant outcomes in patients operated on for knee osteoarthritis with tibial osteotomy. BMC Musculoskelet Disord 2005; 6: 18 .

[61] Rat AC, Guillemin F, Osnowycz G, et al. Total hip or knee replacement for osteoarthritis: mid- and long-term quality of life. Arthritis Care Res (Hoboken) 2010; 62(1): 54-62.

[62] Losina E, Walensky RP, Kessler CL, et al. Cost-effectiveness of total knee arthroplasty in the United States: patient risk and hospital volume. Arch Intern Med 2009; 169(12): 1113-21; discussion 21-2.

[63] Roos EM, Lohmander LS. The Knee injury and Osteoarthritis Outcome Score (KOOS): from joint injury to osteoarthritis. Health Qual Life Outcomes 2003; 1: 64.

[64] Laslett LL, Quinn SJ, Winzenberg TM, Sanderson K, Cicuttini F, Jones G. A prospective study of the impact of musculoskeletal pain and radiographic osteoarthritis on health related quality of life in community dwelling older people. BMC Musculoskelet Disord 2012; 13: 168 . 